their health. But the available answers are rapidly becoming less definitive: the ever-increasing number of genome-wide association studies, which provide a major portion of the genetic markers for disease risks, offer results that even researchers can find hard to interpret, and often flag up markers that are not the most useful predictors of complex traits (see page 712).

This means that customers will frequently receive results telling them only that they face the ambiguous possibility of a somewhat elevated risk of a little-understood disorder. Presumably, most customers in that position will simply be more vigilant about disease screening. But if the ambiguous, slightly elevated risk relates to a frightening condition such as breast cancer, some individuals might feel compelled to undertake drastic and perhaps needless measures, such as prophylactic mastectomy.

The HGC's principles, if adopted, should help to minimize such panic reactions. For example, the HGC advocates that genetic counselling be provided both before and after testing for serious hereditary diseases. But there is room to go further and, on page 724, Craig
Venter and his colleagues offer nine recommendations for how to do so. For example, Venter's team urges companies to agree on a core set of non-ambiguous genetic markers - ones that put a carrier at high risk of developing a specific condition. Companies would be well-advised to follow this recommendation, as such an agreement would help to avoid conflicting messages and inconsistent results across the industry.

Ultimately, however, government regulators may feel compelled to step in on the grounds that industry self-policing no longer offers consumers adequate protection. The US Food and Drug Administration has already recruited bioethicist Alta Charo, in part to advise commissioner Peggy Hamburg on a comprehensive approach to regulating these tests.

Government regulators should proceed with care, given the dizzying speed at which the science of personalized genomics is advancing. But in the interim, DNA-test providers should up their game by providing only clinically useful information and spelling out exactly how much biology remains unknown.

\title{
How to win trust over flu
}

\section{Mass-vaccination campaigns for the pandemic H1N1 virus must take public concerns into account.}

A $s$ countries roll out their campaigns for large-scale vaccination against pandemic H1N1 flu, a poll released last week by the Harvard School of Public Health in Boston, Massachusetts, found that only four in ten US adults have definitely decided to get vaccinated themselves, and just half plan to get the shot for their children (go.nature.com/wiB8V3). Harvard's results parallel those from other surveys, both inside and outside the United States, all of which suggest that many people are still dubious about the vaccine. Public-health authorities, who are keen to contain the pandemic's spread, need to realize that their best hope of dealing with such public reluctance is to patiently address the concerns that underlie it.

Sometimes, it's true, those concerns go beyond any appeal to reason. They grow out of a visceral mistrust of authority in general - and of government, regulatory agencies, medical researchers and multinational pharmaceutical companies, in particular. A sophisticated anti-vaccine movement has emerged that plays on this wariness, and helps to feed the conspiracy theories about the $\mathrm{H} 1 \mathrm{~N} 1$ vaccine that are circulating on the Internet and in viral e-mails.

But far more often, say researchers who have studied this subject, people are assessing vaccination through a perfectly rational costbenefit analysis. There is a widespread public perception, for example, that the vaccine's safety trials have been rushed - the Harvard study found that possible side effects were respondents' main concern - and that $\mathrm{H} 1 \mathrm{~N} 1$ flu is mild. As a result, many feel no urgent need to be vaccinated, preferring to hold off until they see how the first phases of the vaccination programme go. Indeed, the Harvard poll also found that some $60 \%$ of those who don't intend to get a shot are open to changing their mind if people in their community become severely ill or die.

Such deliberations reflect a perfectly legitimate decision-making process, says Peter Sandman, a risk-communication consultant in Princeton, New Jersey. And governments, he advises, should frame their public-education campaigns in ways that respect people's judgement and their wait-and-see attitude. Research in risk communication strongly shows that when over-eager officials pressure members of the public who are already sceptical and ambivalent, while being openly dismissive of public concerns, they only end up stoking resistance.

Instead, officials should focus on providing people with the information they need to make good choices for themselves. This should include reminders that coincidences do happen: in any massvaccination campaign, at least a few people will fall ill immediately after receiving their shot for reasons that have nothing to do with the vaccine - a possibility vividly highlighted last week by the death of a 14-year-old British girl hours after receiving a vaccine against human papilloma virus. Regulatory authorities need to better explain the extensive safety tests that vaccines undergo and, at the same time, build confidence by being utterly transparent in the reporting and investigation of any suspect events linked to vaccination.

The public-education campaign should also correct the misconception that $\mathrm{H} 1 \mathrm{~N} 1 \mathrm{flu}$ is mild. It is mild in most who catch it. But for those individuals - mainly young adults - who will develop the severe form, H1N1 is life-threatening. Moreover, because the virus is new and immunity is lacking, many more people will get it than is typical for seasonal flu, and the toll of serious illness and deaths will accordingly be that much higher.

Finally, people should be reminded that vaccination isn't just about protecting themselves; it's also about not spreading the flu to others, which, importantly, alleviates pressure on overstretched hospitals. Campaigns should give altruistic appeals far more prominence than they typically have in the past; research shows that they can be surprisingly effective.

More generally, for officials and researchers at all levels, the scepticism over the pandemic vaccine should serve as a timely reminder of the imperative to work to gain greater public trust in science-based advice and in those who give it. 